\title{
Conserved currents in gravitational models with quasi-invariant Lagrangians: Application to teleparallel gravity
}

\author{
Yuri N. Obukhov* \\ Instituto de Física Teórica, Universidade Estadual Paulista, Rua Pamplona 145, 01405-900 São Paulo, Brazil \\ and Department of Theoretical Physics, Moscow State University, 117234 Moscow, Russia \\ Guillermo F. Rubilar \\ Instituto de Física Teórica, Universidade Estadual Paulista, Rua Pamplona 145, 01405-900 São Paulo, Brazil \\ and Departamento de Física, Universidad de Concepción, Casilla 160-C, Concepción, Chile \\ J. G. Pereira \\ Instituto de Física Teórica, Universidade Estadual Paulista, Rua Pamplona 145, 01405-900 São Paulo, Brazil
}

(Received 15 September 2006; published 2 November 2006)

\begin{abstract}
Conservation laws in gravitational theories with diffeomorphism and local Lorentz symmetry are studied. Main attention is paid to the construction of conserved currents and charges associated with an arbitrary vector field that generates a diffeomorphism on the spacetime. We further generalize previous results for the case of gravitational models described by quasi-invariant Lagrangians, that is, Lagrangians that change by a total derivative under the action of the local Lorentz group. The general formalism is then applied to the teleparallel models, for which the energy and the angular momentum of a Kerr black hole are calculated. The subsequent analysis of the results obtained demonstrates the importance of the choice of the frame.
\end{abstract}

DOI: 10.1103/PhysRevD.74.104007

PACS numbers: 04.20.Cv, 04.20.Fy, 04.50.+h

\section{INTRODUCTION}

In this paper, we continue the study of conservation laws in gravitational theories with diffeomorphism and local Lorentz symmetries. The previous results, as well as an overview of the earlier literature can be found in $[1,2]$, see also the extensive reference list in [3]. More specifically, we will be interested in the conserved currents and charges associated with vector fields that generate arbitrary diffeomorphisms on the spacetime manifold.

Most gravitational models are normally invariant under both spacetime diffeomorphisms and local Lorentz transformations of the frames. However, the Lagrangian may be shifted by a total derivative term (equivalently, by a boundary term). This actually happens in many gravitational field models: (i) when one adds a noninvariant boundary term to the original Lagrangian, (ii) when the gravitational dynamics is described in the purely tetrad framework [1], (iii) when the Lagrangian includes topological terms (e.g., of the Chern-Simons type [4-6]), typically in 3 and 5 dimensions, for example [7-12].

For models with Lorentz-invariant Lagrangians, we have shown in [2] that it is possible to define invariant conserved currents for every vector field $\xi$. These conserved currents do not depend on the coordinate system or the tetrad frame used to compute them. They depend only on the field configuration and on the choice of the vector field $\xi$. The interpretation of $\xi$ is an important geometrical

\footnotetext{
*Electronic address: yo@ift.unesp.br

${ }^{\dagger}$ Electronic address: grubilar@udec.cl

Electronic address: jpereira@ift.unesp.br
}

and physical issue. For example, when $\xi$ is timelike, the corresponding charge has the meaning of the energy of the gravitating system with respect to an observer moving along the integral lines of $\xi$, with 4-velocity $u=\xi /|\xi|$, cf. [13]. In this way, the dependence of the conserved charges on $\xi$ describes the usual dependence of the energy of a system on the choice and on the dynamics of a physical observer. Furthermore, let us recall that under certain conditions discussed in [2], the matter current $J^{\text {mat }}[\xi]=$ $\left.\xi^{i} T_{i}{ }^{j} \partial_{j}\right\rfloor \eta$ is conserved, with $T_{i}^{j}$ being the energymomentum tensor of matter, and $\eta$ the volume 4-form. The corresponding invariant charge $Q^{\mathrm{mat}}[\xi]=\int_{S} J^{\mathrm{mat}}[\xi]$ is, therefore, the integral of the projection of the energy momentum along the vector $\xi$. The charge $\mathcal{Q}^{\text {mat }}[\xi]$ reduces to the usual expression $\int_{S} T_{0}^{0} \sqrt{-g} d x^{1} \wedge d x^{2} \wedge d x^{3}$ in coordinates adapted to $\xi$ such that $\xi=\partial_{0}$, and the hypersurface $S$ is defined by $x^{0}=$ constant. This general idea of defining the energy of a system as a scalar (i.e., invariant) depending on some vector field is a generalization of the well-known construction for point particles, see, for instance, Sect. 2.8 (and, in particular, Eq. (2.29)) of [14].

In the first part of this paper we generalize the formalism developed in [2], and define conserved currents and charges for models with "quasi-invariant" Lagrangians (that is, Lagrangians that change by a total derivative under local Lorentz transformations). In the second part we apply this formalism to teleparallel gravity. As is well known, in addition to the geometric framework of general relativity, gravitation can also be described in terms of a gauge theory. In fact, teleparallel gravity corresponds to a gauge 
theory for the translation group [15-17]. In this theory, instead of curvature, torsion represents the gravitational field. In spite of this fundamental difference, teleparallel gravity is found to be equivalent to general relativity. This means essentially that the gravitational interaction can be described alternatively in terms of curvature, as is usually done in general relativity, or in terms of torsion, as in teleparallel gravity.

One may wonder why gravitation has two different descriptions. Such a dualism is related to a peculiar property of gravitation, called universality. As is well known, at least at the classical level, particles with different masses and different compositions feel it in such a way that all of them acquire the same acceleration and, given the same initial conditions, follow the same path. Such universality of response is one of the most fundamental characteristics of the gravitational interaction. It is unique, peculiar to gravitation: no other basic interaction of nature has it. And it is exactly this property that makes a geometrized formulation of gravitation possible in addition to the gauge description of teleparallel gravity. As the sole universal interaction, it is the only one to allow a geometrical interpretation, and two alternative descriptions. One may also wonder why it is a gauge theory for the translation group, and not for another spacetime group. The reason for this is related to the source of gravitation, that is, energy and momentum. As is well known from Noether's theorem, these quantities are conserved provided the physical system is invariant under spacetime translations. It is then natural to expect that the gravitational field will be associated to the translation group. This is quite similar to the electromagnetic field, whose source-the electric fourcurrent-is conserved due to invariance of the theory under transformations of the unitary group $U(1)$, the gauge group of Maxwell's theory.

Our general notations are the same as in [18]. In particular, we use the Latin indices $i, j, \ldots$ for local holonomic spacetime coordinates and the Greek indices $\alpha, \beta, \ldots$ label (co)frame components. Particular frame components are denoted by hats, $\hat{0}, \hat{1}$, etc. As usual, the exterior product is denoted by $\wedge$, while the interior product of a vector $\xi$ and a $p$-form $\Psi$ is denoted by $\xi\rfloor \Psi$. The vector basis dual to the frame 1-forms $\vartheta^{\alpha}$ is denoted by $e_{\alpha}$ and they satisfy $\left.e_{\alpha}\right\rfloor \vartheta^{\beta}=\delta_{\alpha}^{\beta}$. Using local coordinates $x^{i}$, we have $\vartheta^{\alpha}=$ $h_{i}^{\alpha} d x^{i}$ and $e_{\alpha}=h_{\alpha}^{i} \partial_{i}$. We define the volume $n$-form by $\eta:=\vartheta^{\hat{0}} \wedge \cdots \wedge \vartheta^{\hat{n}}$. Furthermore, with the help of the interior product we define $\left.\left.\eta_{\alpha}:=e_{\alpha}\right\rfloor \eta, \eta_{\alpha \beta}:=e_{\beta}\right\rfloor \eta_{\alpha}$, $\left.\eta_{\alpha \beta \gamma}:=e_{\gamma}\right\rfloor \eta_{\alpha \beta}$, etc., which are bases for $(n-1)$-, $(n-2)-$, and $(n-3)$-forms, etc., respectively. Finally, $\left.\eta_{\alpha_{1} \cdots \alpha_{n}}=e_{\alpha_{n}}\right\rfloor \eta_{\alpha_{1} \cdots \alpha_{n-1}}$ is the Levi-Civita tensor density. The $\eta$-forms satisfy the identities:

$$
\begin{gathered}
\vartheta^{\beta} \wedge \eta_{\alpha}=\delta_{\alpha}^{\beta} \eta, \\
\vartheta^{\beta} \wedge \eta_{\mu \nu}=\delta_{\nu}^{\beta} \eta_{\mu}-\delta_{\mu}^{\beta} \eta_{\nu},
\end{gathered}
$$

$$
\begin{aligned}
\vartheta^{\beta} \wedge \eta_{\alpha \mu \nu}= & \delta_{\alpha}^{\beta} \eta_{\mu \nu}+\delta_{\mu}^{\beta} \eta_{\nu \alpha}+\delta_{\nu}^{\beta} \eta_{\alpha \mu}, \\
\vartheta^{\beta} \wedge \eta_{\alpha \gamma \mu \nu}= & \delta_{\nu}^{\beta} \eta_{\alpha \gamma \mu}-\delta_{\mu}^{\beta} \eta_{\alpha \gamma \nu}+\delta_{\gamma}^{\beta} \eta_{\alpha \mu \nu} \\
& -\delta_{\alpha}^{\beta} \eta_{\gamma \mu \nu},
\end{aligned}
$$

etc. The line element $d s^{2}=g_{\alpha \beta} \vartheta^{\alpha} \otimes \vartheta^{\beta}$ is defined by the spacetime metric $g_{\alpha \beta}$ of signature $(+,-, \cdots,-)$.

\section{THE LAGRANGE-NOETHER MACHINERY}

The gravitational field is described by the coframe $\vartheta^{\alpha}$ and the Lorentz connection $\Gamma_{\alpha}{ }^{\beta}$ 1-forms. The matter fields $\Psi^{A}$ can be Lorentz-covariant forms of an arbitrary rank $p$.

Let $V^{\text {tot }}=V^{\text {tot }}\left(\vartheta, d \vartheta, \Gamma, d \Gamma, \Psi^{A}, d \Psi^{A}\right)$ be an arbitrary Lagrangian. The total variation then formally reads as (cf. [2]):

$$
\begin{aligned}
\delta V^{\mathrm{tot}}= & \delta \vartheta^{\alpha} \wedge \mathcal{F}_{\alpha}+\delta \Gamma_{\alpha}{ }^{\beta} \wedge \mathcal{F}^{\alpha}{ }_{\beta}+\delta \Psi^{A} \wedge \mathcal{F}_{A} \\
& -d\left(\delta \vartheta^{\alpha} \wedge \mathcal{H}_{\alpha}+\delta \Gamma_{\alpha}{ }^{\beta} \wedge \mathcal{H}^{\alpha}{ }_{\beta}+\delta \Psi^{A} \wedge \mathcal{H}_{A}\right)
\end{aligned}
$$

Here as usual we introduce the generalized (translational, rotational, and matter) field momenta by

$$
\begin{gathered}
\mathcal{H}_{\alpha}:=-\frac{\partial V^{\mathrm{tot}}}{\partial d \vartheta^{\alpha}}, \quad \mathcal{H}_{\beta}^{\alpha}{ }_{\beta}:=-\frac{\partial V^{\mathrm{tot}}}{\partial d \Gamma_{\alpha}^{\beta}}, \\
\mathcal{H}_{A}:=-\frac{\partial V^{\mathrm{tot}}}{\partial d \Psi^{A}},,
\end{gathered}
$$

whereas the variational derivatives with respect to (w.r.t.) the fields are defined by

$$
\begin{gathered}
\mathcal{F}_{\alpha}:=\frac{\delta V^{\mathrm{tot}}}{\delta \vartheta^{\alpha}}=\frac{\partial V^{\mathrm{tot}}}{\partial \vartheta^{\alpha}}-d \mathcal{H}_{\alpha}, \\
\mathcal{F}_{\beta}^{\alpha}:=\frac{\delta V^{\mathrm{tot}}}{\delta \Gamma_{\alpha}{ }^{\beta}}=\frac{\partial V^{\mathrm{tot}}}{\partial \Gamma_{\alpha}{ }^{\beta}}-d \mathcal{H}^{\alpha}{ }_{\beta}, \\
\mathcal{F}_{A}:=\frac{\delta L}{\delta \Psi^{A}}=\frac{\partial V^{\mathrm{tot}}}{\partial \Psi^{A}}+(-1)^{p} d \mathcal{H}_{A} .
\end{gathered}
$$

\section{A. Lagrangians for gravity and matter: field equations}

The total Lagrangian is a sum $V^{\text {tot }}=V+L$ of the gravitational Lagrangian $V=V(\vartheta, d \vartheta, \Gamma, d \Gamma)$ and the matter Lagrangian $L=L\left(\Psi^{A}, d \Psi^{A}, \vartheta, d \vartheta, \Gamma, d \Gamma\right)$. The dependence of the latter on the derivatives of the gravitational potentials may arise for models with nonminimal coupling. Then one usually defines for the gravitational Lagrangian the derivatives:

$$
\begin{gathered}
\mathcal{E}_{\alpha}:=\frac{\delta V}{\delta \vartheta^{\alpha}}=-d H_{\alpha}+\frac{\partial V}{\partial \vartheta^{\alpha}}, \\
\mathcal{C}_{\beta}^{\alpha}:=\frac{\delta V}{\delta \Gamma_{\alpha}{ }^{\beta}}=-d H^{\alpha}{ }_{\beta}+\frac{\partial V}{\partial \Gamma_{\alpha}{ }^{\beta}} .
\end{gathered}
$$


Here $H_{\alpha}$ and $H_{\beta}^{\alpha}$ are defined analogously to (2.2), but for the gravitational Lagrangian $V$. These quantities describe the left-hand (geometric) sides of the gravitational field equations. Analogous variational derivatives of the matter Lagrangian define the energy momentum and spin of matter:

$$
\Sigma_{\alpha}:=\frac{\delta L}{\delta \vartheta^{\alpha}}, \quad \tau_{\beta}^{\alpha}:=\frac{\delta L}{\delta \Gamma_{\alpha}{ }^{\beta}} .
$$

The total system of coupled field equations then reads

$$
\begin{gathered}
\mathcal{F}_{\alpha}=\mathcal{E}_{\alpha}+\Sigma_{\alpha}=0, \\
\mathcal{F}_{\beta}^{\alpha}=\mathcal{C}_{\beta}^{\alpha}+\tau_{\beta}^{\alpha}=0, \\
\mathcal{F}_{A}=0 .
\end{gathered}
$$

\section{B. Noether identities for the Lorentz symmetry}

Let us assume that for an infinitesimal Lorentz transformation,

$$
\begin{gathered}
\delta \vartheta^{\alpha}=\varsigma \varepsilon^{\alpha}{ }_{\beta} \vartheta^{\beta}, \quad \delta \Gamma_{\beta}{ }^{\alpha}=-\varsigma D \varepsilon^{\alpha}{ }_{\beta}, \\
\delta \Psi^{A}=\varsigma \varepsilon^{\alpha}{ }_{\beta}\left(\rho^{\beta}{ }_{\alpha}\right)_{B}^{A}{ }_{B} \Psi^{B},
\end{gathered}
$$

with $\Lambda_{\beta}^{\alpha}=\delta_{\beta}^{\alpha}+\varsigma \varepsilon_{\beta}^{\alpha}, \varepsilon_{\alpha \beta}=-\varepsilon_{\beta \alpha}$, and $\varsigma$ an infinitesimal constant parameter $\left(\rho^{\beta}{ }_{\alpha}\right.$ are the Lorentz generators for the matter fields), the Lagrangian is changed by a total derivative:

$$
\delta_{\varepsilon} V^{\mathrm{tot}}=-d\left(\mathrm{~s}^{\alpha}{ }_{\beta} v_{\alpha}^{\beta}\right) .
$$

Here $v_{\alpha}^{\beta}$ is some $(n-1)$-form. In view of the skew symmetry of the Lorentz parameters, it is also antisymmetric, $v_{\alpha \beta}=-v_{\beta \alpha}$.

Then (2.1) yields straightforwardly

$$
\begin{aligned}
\varepsilon^{\alpha}{ }_{\beta}\left(\vartheta^{\beta}\right. & \left.\wedge \mathcal{F}_{\alpha}+D \mathcal{F}^{\beta}{ }_{\alpha}+\left(\rho^{\beta}{ }_{\alpha}\right)^{A}{ }_{B} \Psi^{B} \wedge \mathcal{F}_{A}\right) \\
+d\left[\varepsilon ^ { \alpha } { } _ { \beta } \left(v^{\beta}{ }_{\alpha}-\mathcal{C}^{\beta}{ }_{\alpha}-\vartheta^{\beta}\right.\right. & \wedge \mathcal{H}_{\alpha}-D \mathcal{H}^{\beta}{ }_{\alpha} \\
& \left.\left.\quad-\left(\rho^{\beta}{ }_{\alpha}\right)^{A}{ }_{B} \Psi^{B} \wedge \mathcal{H}_{A}\right)\right]=0 .
\end{aligned}
$$

Notice that the covariant exterior derivative $D:=d+\rho^{\beta}{ }_{\alpha}$ is used only as an abbreviation when acting on $\mathcal{H}^{\beta}{ }_{\alpha}$ and $\varepsilon^{\alpha}{ }_{\beta}$ in (2.12), since these quantities are not, in general, Lorentz-covariant fields.

As a result, we find the two Noether identities (since the transformation parameters $\varepsilon$ and their derivatives $d \varepsilon$ are pointwise arbitrary):

$$
\begin{gathered}
D \mathcal{F}_{\alpha \beta}+\vartheta_{[\alpha} \wedge \mathcal{F}_{\beta]}+\left(\rho_{\alpha}^{\beta}\right)_{B}^{A}{ }_{B} \Psi^{B} \wedge \mathcal{F}_{A} \equiv 0, \\
v_{\alpha \beta}-\mathcal{F}_{\alpha \beta}-\vartheta_{[\alpha} \wedge \mathcal{H}_{\beta]}-D \mathcal{H}_{\alpha \beta} \\
-\left(\rho_{\alpha \beta}\right)_{B}^{A} \Psi^{B} \wedge \mathcal{H}_{A} \equiv 0
\end{gathered}
$$

The second relation is trivial in models with invariant Lagrangians.

\section{Noether identities for the diffeomorphism symmetry}

Let us derive the consequences of the assumed diffeomorphism invariance of $V^{\text {tot }}$. Let $f$ be an arbitrary local diffeomorphism on the spacetime manifold. It acts with the pullback map $f^{*}$ on all the geometrical quantities, and the invariance of the theory means that $V^{\mathrm{tot}}\left(f^{*} \vartheta, f^{*} d \vartheta, f^{*} \Gamma, f^{*} d \Gamma, f^{*} \Psi, f^{*} d \Psi\right)=$

$f^{*}\left(V^{\text {tot }}(\vartheta, d \vartheta, \Gamma, d \Gamma, \Psi, d \Psi)\right)$. Consider an arbitrary vector field $\xi$ and the corresponding local 1-parameter group of diffeomorphisms $f_{t}$ generated along this vector field. Then, using $f_{t}$ in the above formula and differentiating w.r.t. the parameter $t$, we find the identity

$$
\begin{aligned}
\left(\ell_{\xi} \vartheta^{\alpha}\right) \wedge \frac{\partial V^{\mathrm{tot}}}{\partial \vartheta^{\alpha}}+ & \left(\ell_{\xi} \Gamma_{\alpha}{ }^{\beta}\right) \wedge \frac{\partial V^{\mathrm{tot}}}{\partial \Gamma_{\alpha}{ }^{\beta}}+\left(\ell_{\xi} \Psi^{A}\right) \wedge \frac{\partial V^{\mathrm{tot}}}{\partial \Psi^{A}} \\
& -\left(\ell_{\xi} d \vartheta^{\alpha}\right) \wedge \mathcal{H}_{\alpha}-\left(\ell_{\xi} d \Gamma_{\alpha}{ }^{\beta}\right) \wedge \mathcal{H}^{\alpha}{ }_{\beta} \\
& -\left(\ell_{\xi} d \Psi^{A}\right) \wedge \mathcal{H}_{A}=\ell_{\xi} V^{\mathrm{tot}} .
\end{aligned}
$$

Moving the last term to the left-hand side (1.h.s.), and using the Lie derivative (that is given on exterior forms by $\ell_{\xi}=$ $d \xi\rfloor+\xi\rfloor d$ ), we then find the identity $A+d B=0$ with

$$
\begin{aligned}
A= & \left.\xi^{\alpha}\left(-d \mathcal{F}_{\alpha}-e_{\alpha}\right\rfloor \Gamma_{\gamma}{ }^{\beta} d \mathcal{F}^{\gamma}{ }_{\beta}+e_{\alpha}\right\rfloor d \vartheta^{\beta} \wedge \mathcal{F}_{\beta} \\
& \left.\left.+e_{\alpha}\right\rfloor d \Gamma_{\gamma}{ }^{\beta} \wedge \mathcal{F}^{\gamma}{ }_{\beta}+(-1)^{p} e_{\alpha}\right\rfloor \Psi^{A} \wedge d \mathcal{F}_{A} \\
& \left.\left.+e_{\alpha}\right\rfloor d \Psi^{A} \wedge \mathcal{F}_{A}\right),
\end{aligned}
$$

$$
\begin{aligned}
B= & \left.\xi^{\alpha}\left(\frac{\partial V^{\text {tot }}}{\partial \vartheta^{\alpha}}+e_{\alpha}\right\rfloor \Gamma_{\beta} \gamma \frac{\partial V^{\text {tot }}}{\partial \Gamma_{\beta}^{\gamma}}+e_{\alpha}\right\rfloor \Psi^{A} \wedge \frac{\partial V^{\text {tot }}}{\partial \Psi^{A}} \\
& \left.\left.-e_{\alpha}\right\rfloor d \vartheta^{\beta} \wedge \mathcal{H}_{\beta}-e_{\alpha}\right\rfloor d \Gamma_{\gamma}{ }^{\beta} \wedge \mathcal{H}^{\gamma}{ }_{\beta} \\
& \left.\left.\left.-e_{\alpha}\right\rfloor d \Psi^{A} \wedge \mathcal{H}_{A}-e_{\alpha}\right\rfloor V^{\text {tot }}\right) .
\end{aligned}
$$

Since the diffeomorphism invariance holds for arbitrary vector fields $\xi$, then $A$ and $B$ must necessarily vanish, and we find the two Noether identities:

$$
\begin{aligned}
& \left.\left.d\left(\mathcal{F}_{\alpha}+e_{\alpha}\right\rfloor \Gamma_{\gamma}{ }^{\beta} \mathcal{F}_{\beta}^{\gamma}+e_{\alpha}\right\rfloor \Psi^{A} \wedge \mathcal{F}_{A}\right) \\
& \quad \equiv\left(\ell_{e_{\alpha}} \vartheta^{\beta}\right) \wedge \mathcal{F}_{\beta}+\left(\ell_{e_{\alpha}} \Gamma_{\gamma}{ }^{\beta}\right) \wedge \mathcal{F}_{\beta}^{\gamma}+\left(\ell_{e_{\alpha}} \Psi^{A}\right) \wedge \mathcal{F}_{A},
\end{aligned}
$$

$$
\begin{aligned}
\frac{\partial V^{\text {tot }}}{\partial \vartheta^{\alpha}}+ & \left.\left.e_{\alpha}\right\rfloor \Gamma_{\beta} \gamma \frac{\partial V^{\text {tot }}}{\partial \Gamma_{\beta}^{\gamma}}+e_{\alpha}\right\rfloor \Psi^{A} \wedge \frac{\partial V^{\text {tot }}}{\partial \Psi^{A}} \\
\equiv & \left.\left.\left.e_{\alpha}\right\rfloor V^{\text {tot }}+e_{\alpha}\right\rfloor d \vartheta^{\beta} \wedge \mathcal{H}_{\beta}+e_{\alpha}\right\rfloor d \Gamma_{\gamma}{ }^{\beta} \wedge \mathcal{H}^{\gamma}{ }_{\beta} \\
& \left.+e_{\alpha}\right\rfloor d \Psi^{A} \wedge \mathcal{H}_{A} .
\end{aligned}
$$




\section{CURRENT ASSOCIATED WITH A VECTOR FIELD}

We define the current $(n-1)$-form by

$$
\begin{aligned}
J[\xi, \varepsilon]:= & \xi\rfloor V^{\text {tot }}-\varepsilon^{\alpha}{ }_{\beta} v^{\beta}{ }_{\alpha}+\mathcal{L}_{\{\xi, \varepsilon\}} \vartheta^{\alpha} \wedge \mathcal{H}_{\alpha} \\
& +\mathcal{L}_{\{\xi, \varepsilon\}} \Gamma_{\alpha}{ }^{\beta} \wedge \mathcal{H}^{\alpha}{ }_{\beta}+\mathcal{L}_{\{\xi, \varepsilon\}} \Psi^{A} \wedge \mathcal{H}_{A} .
\end{aligned}
$$

Here we introduced the "generalized Lie derivatives" of the gravitational fields by

$$
\begin{array}{r}
\mathcal{L}_{\{\xi, \varepsilon\}} \vartheta^{\alpha}:=\ell_{\xi} \vartheta^{\alpha}+\varepsilon^{\alpha}{ }_{\beta} \vartheta^{\beta}, \\
\mathcal{L}_{\{\xi, \varepsilon\}} \Gamma_{\alpha}{ }^{\beta}:=\ell_{\xi} \Gamma_{\alpha}{ }^{\beta}-D \varepsilon^{\beta}{ }_{\alpha}, \\
\mathcal{L}_{\{\xi, \varepsilon\}} \Psi^{A}:=\ell_{\xi} \Psi^{A}+\varepsilon^{\alpha}{ }_{\beta}\left(\rho^{\beta}{ }_{\alpha}\right)^{A}{ }_{B} \Psi^{B} .
\end{array}
$$

The current (3.1) satisfies the identity

$$
\begin{aligned}
d J[\xi, \varepsilon]= & \mathcal{L}_{\{\xi, \varepsilon\}} \vartheta^{\alpha} \wedge \mathcal{F}_{\alpha}+\mathcal{L}_{\{\xi, \varepsilon\}} \Gamma_{\alpha}{ }^{\beta} \wedge \mathcal{F}^{\alpha}{ }_{\beta} \\
& +\mathcal{L}_{\{\xi, \varepsilon\}} \Psi^{A} \wedge \mathcal{F}_{A} .
\end{aligned}
$$

This is just the total variation (2.1) in a different disguise.

Using Eqs. (3.2), (3.3), and (3.4), the definitions (2.6) and (2.7), and the Noether identities (2.16) and (2.21), we can identically rewrite the current (3.1) in the form

$$
\begin{aligned}
J[\xi, \varepsilon]= & \left.d\left(\xi^{\alpha} \mathcal{H}_{\alpha}+\Xi_{\alpha}{ }^{\beta}[\xi, \varepsilon] \mathcal{H}^{\alpha}{ }_{\beta}+\xi\right\rfloor \Psi^{A} \wedge \mathcal{H}_{A}\right) \\
& \left.+\xi^{\alpha} \mathcal{F}_{\alpha}+\Xi_{\alpha}{ }^{\beta}[\xi, \varepsilon] \mathcal{F}^{\alpha}{ }_{\beta}+\xi\right] \Psi^{A} \wedge \mathcal{F}_{A},
\end{aligned}
$$

where we introduced the notation

$$
\left.\Xi_{\alpha}{ }^{\beta}[\xi, \varepsilon]:=\xi\right] \Gamma_{\alpha}{ }^{\beta}-\varepsilon^{\beta}{ }_{\alpha} .
$$

\section{A. Gravitational current for Yano-Lie derivative}

There exist different choices of $\varepsilon^{\alpha}{ }_{\beta}(\xi)$ for a given vector field $\xi$, that correspond to the use of different generalized Lie derivatives [2]. They give rise to different conserved currents and charges. The "minimal" choice [2]

$$
\left.\varepsilon_{\alpha \beta}=-\Theta_{\alpha \beta}=-e_{[\alpha}\right\rfloor \ell_{\xi} \vartheta_{\beta]}
$$

defines the generalized Lie derivative (which we denote $\mathcal{L}_{\xi}$ ) in the sense of Yano [19]. We will work with this choice for the rest of the paper.

Now, let us consider the case in which we take $V^{\text {tot }}=V$, i.e. the Lagrangian of the gravitational field. In this case, the conserved current for an arbitrary quasi-invariant gravitational Lagrangian is found to be

$$
\begin{aligned}
\mathcal{J}^{\operatorname{grav}}[\xi]:= & \xi\rfloor V-\Theta^{\alpha \beta} v_{\alpha \beta}+\mathcal{L}_{\xi} \vartheta^{\alpha} \wedge H_{\alpha} \\
& +\mathcal{L}_{\xi} \Gamma_{\alpha}{ }^{\beta} \wedge H^{\alpha}{ }_{\beta} .
\end{aligned}
$$

In accordance with the above derivations, it satisfies

$$
d \mathcal{J}^{\text {grav }}[\xi]=\mathcal{L}_{\xi} \vartheta^{\alpha} \wedge \mathcal{E}_{\alpha}+\mathcal{L}_{\xi} \Gamma_{\alpha}{ }^{\beta} \wedge \mathcal{C}^{\alpha}{ }_{\beta},
$$

and it can be identically recast in the form

$$
\mathcal{J}^{\operatorname{grav}}[\xi]=d\left(\xi^{\alpha} H_{\alpha}+\Xi_{\alpha}{ }^{\beta} H^{\alpha}{ }_{\beta}\right)+\xi^{\alpha} \mathcal{E}_{\alpha}+\Xi_{\alpha}{ }^{\beta} \mathcal{C}^{\alpha}{ }_{\beta},
$$

where now $\left.\Xi_{\alpha}{ }^{\beta}=\xi\right\rfloor \Gamma_{\alpha}{ }^{\beta}+\Theta_{\alpha}{ }^{\beta}$.

\section{B. Matter current}

In an analogous way, if we consider the case when $V^{\text {tot }}=L$, we can derive the current for matter. Usually, matter is described by Lorentz-covariant (scalar or spinor) fields that are coupled to gravity in a general coordinateand local Lorentz-covariant manner (in accordance with the covariance and equivalence principles). Taking this into account, we conclude that the matter Lagrangian is constructed only from the covariant objects, i.e., $L=$ $L(\Psi, D \Psi, T, R)$. Thus, the matter Lagrangian is invariant under the local Lorentz group, and the matter current can be written as in [2]:

$$
\begin{aligned}
\mathcal{J}^{\mathrm{mat}}[\xi]:= & \xi] L-\mathcal{L}_{\xi} \vartheta^{\alpha} \wedge \frac{\partial L}{\partial T^{\alpha}}-\mathcal{L}_{\xi} \Gamma_{\alpha}{ }^{\beta} \wedge \frac{\partial L}{\partial R_{\alpha}{ }^{\beta}} \\
& -\mathcal{L}_{\xi} \Psi^{A} \wedge \frac{\partial L}{\partial D \Psi^{A}} .
\end{aligned}
$$

This current satisfies

$$
\begin{aligned}
d \mathcal{J}^{\text {mat }}[\xi]= & \mathcal{L}_{\xi} \vartheta^{\alpha} \wedge \Sigma_{\alpha}+\mathcal{L}_{\xi} \Gamma_{\alpha}{ }^{\beta} \wedge \tau_{\beta}^{\alpha}{ }_{\beta} \\
& +\mathcal{L}_{\xi} \Psi^{A} \wedge \frac{\delta L}{\delta \Psi^{A}} .
\end{aligned}
$$

Furthermore, one can verify that

$$
\begin{aligned}
\mathcal{J}^{\mathrm{mat}}[\xi]= & \left.-d\left(\xi^{\alpha} \frac{\partial L}{\partial T^{\alpha}}+\Xi_{\alpha}{ }^{\beta} \frac{\partial L}{\partial R_{\alpha}{ }^{\beta}}+\xi\right\rfloor \Psi^{A} \wedge \frac{\partial L}{\partial \Psi^{A}}\right) \\
& \left.+\xi^{\alpha} \Sigma_{\alpha}+\Xi_{\alpha}{ }^{\beta} \tau^{\alpha}{ }_{\beta}+\xi\right\rfloor \Psi^{A} \wedge \frac{\delta L}{\delta \Psi^{A}}
\end{aligned}
$$

\section{Total current}

Let us now consider the total Lagrangian $V^{\text {tot }}=V+L$ of the interacting gravitational and matter fields. The total current is then the sum of the gravitational and the matter currents, given, respectively, by Eqs. (3.9) and (3.12):

$$
\begin{aligned}
\mathcal{J}[\xi]= & \mathcal{J}^{\text {grav }}[\xi]+\mathcal{J}^{\text {mat }}[\xi] \\
= & \xi](V+L)-\Theta^{\alpha \beta} v_{\alpha \beta}-\mathcal{L}_{\xi} \Psi^{A} \wedge \frac{\partial L}{\partial D \Psi^{A}} \\
& +\mathcal{L}_{\xi} \vartheta^{\alpha} \wedge\left(H_{\alpha}-\frac{\partial L}{\partial T^{\alpha}}\right) \\
& +\mathcal{L}_{\xi} \Gamma_{\alpha}{ }^{\beta} \wedge\left(H^{\alpha}{ }_{\beta}-\frac{\partial L}{\partial R_{\alpha}{ }^{\beta}}\right) .
\end{aligned}
$$

The total current, see (3.5), satisfies 


$$
\begin{aligned}
d \mathcal{J}[\xi]= & \mathcal{L}_{\xi} \vartheta^{\alpha} \wedge\left(\mathcal{E}_{\alpha}+\Sigma_{\alpha}\right)+\mathcal{L}_{\xi} \Gamma_{\alpha}{ }^{\beta} \wedge\left(\mathcal{C}^{\alpha}{ }_{\beta}+\tau^{\alpha}{ }_{\beta}\right) \\
& +\mathcal{L}_{\xi} \Psi^{A} \wedge \frac{\delta L}{\delta \Psi^{A}} .
\end{aligned}
$$

Accordingly, when the gravitational and matter fields satisfy the field equations (2.9), (2.10), and (2.11), we obtain a conserved current: $d \mathcal{J}[\xi]=0$. In addition, from (3.11) and (3.14), or directly from (3.6), we find

$$
\begin{aligned}
\mathcal{J}[\xi]= & d\left[\xi^{\alpha}\left(H_{\alpha}-\frac{\partial L}{\partial T^{\alpha}}\right)+\Xi_{\alpha}{ }^{\beta}\left(H^{\alpha}{ }_{\beta}-\frac{\partial L}{\partial R_{\alpha}{ }^{\beta}}\right)\right. \\
& \left.+\xi] \Psi^{A} \wedge \frac{\partial L}{\partial \Psi^{A}}\right]+\xi^{\alpha}\left(\mathcal{E}_{\alpha}+\Sigma_{\alpha}\right) \\
& +\Xi_{\alpha}{ }^{\beta}\left(\mathcal{C}^{\alpha}{ }_{\beta}+\tau^{\alpha}{ }_{\beta}\right) .
\end{aligned}
$$

Thus, the conserved total current is expressed in terms of the superpotential 2-form "on-shell." As a result, the conserved charge is then computed as an integral over the spatial boundary $\partial S$ :

$$
\begin{aligned}
\mathcal{Q}[\xi]:= & \int_{S} \mathcal{J}[\xi] \\
= & \int_{\partial S}\left[\xi^{\alpha}\left(H_{\alpha}-\frac{\partial L}{\partial T^{\alpha}}\right)+\Xi_{\alpha}{ }^{\beta}\left(H^{\alpha}{ }_{\beta}-\frac{\partial L}{\partial R_{\alpha}{ }^{\beta}}\right)\right. \\
& \left.+\xi\rfloor \Psi^{A} \wedge \frac{\partial L}{\partial \Psi^{A}}\right] .
\end{aligned}
$$

This result generalizes the construction of the conserved currents and charges, developed in [2], to the case of the quasi-invariant models. Although the final formulas above and in [2] appear to be identical, there is an essential difference. For the theories with invariant Lagrangians, the gravitational field momenta are defined by $H_{\alpha}=$ $-\partial V / \partial T^{\alpha}$ and $H^{\alpha}{ }_{\beta}=-\partial V / \partial R_{\alpha}{ }^{\beta}$. They are, by construction, covariant under local Lorentz transformations. This fact then guarantees that the conserved current and charge are true scalars under both diffeomorphisms and the local Lorentz group. In contrast, for the quasi-invariant models under consideration, the gravitational field momenta (2.2) are no longer covariant under local Lorentz transformations, not even for the choice of the Lie derivatives in the sense of Yano. As a result, both the conserved current (3.15) and the corresponding charge (3.18) are invariant under diffeomorphisms, but neither of them is a scalar under the local Lorentz group. This fact obviously represents a problem for the physical interpretation of the resulting conserved quantities.

\section{TETRAD FORMULATION OF GRAVITY THEORY}

In order to demonstrate how the general formalism works in physically interesting situations, we will now analyze the 4-dimensional teleparallel gravity theory in the so-called pure tetrad formulation. This model is described by the Lagrangian [1]:

$$
\tilde{V}(\vartheta, d \vartheta)=-\frac{1}{2 \kappa} F^{\alpha} \wedge^{\star}\left({ }^{(1)} F_{\alpha}-2^{(2)} F_{\alpha}-\frac{1}{2}{ }^{(3)} F_{\alpha}\right) .
$$

Here $\kappa=8 \pi G / c^{3}$, and ${ }^{\star}$ denotes the Hodge dual defined by the Minkowski metric $g_{\alpha \beta}=o_{\alpha \beta}:=$ $\operatorname{diag}(+1,-1,-1,-1)$. The latter is used also to raise and lower the Greek (local frame) indices. The 2-form $F^{\alpha}:=$ $d \vartheta^{\alpha}$ is the translational gauge field strength (which in geometric terms is equal to the anholonomity object of the tetrad). The irreducible decomposition of the field strength reads (see [15-17,20] for details)

$$
\begin{aligned}
{ }^{(1)} F^{\alpha} & :=F^{\alpha}-{ }^{(2)} F^{\alpha}-{ }^{(3)} F^{\alpha}, \\
{ }^{(2)} F^{\alpha} & \left.:=\frac{1}{3} \vartheta^{\alpha} \wedge\left(e_{\beta}\right\rfloor F^{\beta}\right), \\
{ }^{(3)} F^{\alpha} & \left.:=\frac{1}{3} e^{\alpha}\right\rfloor\left(\vartheta^{\beta} \wedge F_{\beta}\right) .
\end{aligned}
$$

The variation of the total Lagrangian $V^{\text {tot }}=\tilde{V}+L$ with respect to the tetrad yields the gravitational field equations

$$
d \tilde{H}_{\alpha}-\tilde{E}_{\alpha}=\Sigma_{\alpha} .
$$

Here, in accordance with the general Lagrange-Noether scheme (see Sec. II, and Refs. $[1,15,18]$ ), we have

$$
\begin{gathered}
\tilde{H}_{\alpha}=-\frac{\partial \tilde{V}}{\partial F^{\alpha}}=\frac{1}{2 \kappa} \tilde{\Gamma}^{\beta \gamma} \wedge \eta_{\alpha \beta \gamma}, \\
\left.\left.\tilde{E}_{\alpha}=\frac{\partial \tilde{V}}{\partial \vartheta^{\alpha}}=e_{\alpha}\right\rfloor \tilde{V}+\left(e_{\alpha}\right\rfloor F^{\beta}\right) \wedge \tilde{H}_{\beta} .
\end{gathered}
$$

The teleparallel model (4.1) belongs to the class of quasiinvariant theories. In fact, one can verify that under a change of the coframe $\vartheta^{\prime \alpha}=\Lambda^{\alpha}{ }_{\beta}(x) \vartheta^{\beta}$, the Lagrangian changes by a total derivative:

$$
\tilde{V}\left(\vartheta^{\prime}\right)=\tilde{V}(\vartheta)-\frac{1}{2 \kappa} d\left[\left(\Lambda^{-1}\right)_{\gamma}^{\beta} d \Lambda_{\alpha}^{\gamma} \wedge \eta_{\beta}^{\alpha}\right] .
$$

Hence, for this model we explicitly find $v^{\alpha}{ }_{\beta}=-\frac{1}{2 \kappa} d \eta^{\alpha}{ }_{\beta}$. The field equations (4.5) are, however, Lorentz-covariant. As is well known, they coincide with the usual Einstein equations of general relativity theory. For this reason, the model (4.1) is usually called the teleparallel equivalent of general relativity.

In [1], we studied the transformation laws of the main objects in the tetrad formulation of gravity. In addition to (4.8), one can verify that

$$
\begin{aligned}
\tilde{E}_{\alpha}^{\prime}\left(\vartheta^{\prime}\right)= & \left(\Lambda^{-1}\right)_{\alpha}^{\beta} \tilde{E}_{\beta}(\vartheta)+d\left(\Lambda^{-1}\right)^{\beta}{ }_{\alpha} \wedge \tilde{H}_{\beta} \\
& -\frac{1}{2 \kappa} d\left[\left(\Lambda^{-1}\right)^{\beta}{ }_{\alpha}\left(\Lambda^{-1}\right)^{\nu}{ }_{\gamma} d \Lambda^{\gamma}{ }_{\mu} \wedge \eta_{\beta}{ }^{\mu}{ }_{\nu}\right],
\end{aligned}
$$




$$
\begin{aligned}
\tilde{H}_{\alpha}^{\prime}\left(\vartheta^{\prime}\right)= & \left(\Lambda^{-1}\right)^{\beta}{ }_{\alpha} \tilde{H}_{\beta}(\vartheta)-\frac{1}{2 \kappa}\left(\Lambda^{-1}\right)_{\alpha}^{\beta}\left(\Lambda^{-1}\right)^{\nu}{ }_{\gamma} d \Lambda^{\gamma}{ }_{\mu} \\
& \wedge \eta_{\beta}{ }^{\mu}{ }_{\nu} .
\end{aligned}
$$

\section{A. Conserved charge in the tetrad gravity}

Using the general results of the previous section, we can write down the conserved charge in the tetrad gravity theory as

$$
\tilde{Q}[\xi, \vartheta]=\int_{\partial S} \xi^{\alpha} \tilde{H}_{\alpha}=\frac{1}{2 \kappa} \int_{\partial S} \xi^{\alpha} \tilde{\Gamma}^{\beta \gamma} \wedge \eta_{\alpha \beta \gamma} .
$$

This quantity substantially depends on the choice of the tetrad field configuration $\vartheta$. More exactly, under a local Lorentz transformation, from (4.10) we find

$$
\begin{aligned}
\widetilde{\mathcal{Q}}^{\prime}\left[\xi, \vartheta^{\prime}\right]= & \widetilde{\mathcal{Q}}[\xi, \vartheta]-\frac{1}{2 \kappa} \int_{\partial S} \xi^{\alpha}\left(\Lambda^{-1}\right)^{\nu}{ }_{\gamma} d \Lambda^{\gamma}{ }_{\mu} \\
& \wedge \eta_{\alpha}{ }^{\mu}{ }_{\nu} .
\end{aligned}
$$

Note that the vector field $\xi$ does not depend on the choice of the frame, but its components $\left.\xi^{\alpha}=\xi\right\rfloor \vartheta^{\alpha}$ transform as a vector.

\section{B. Charges for the Kerr solution}

Let us find the conserved charges for a particular tetrad configuration that describes an asymptotically flat axisymmetric rotating vacuum solution: the Kerr solution. We choose the Boyer-Lindquist local coordinate system $(t, r, \theta, \varphi)$, and write the coframe as

$$
\begin{gathered}
\vartheta^{\hat{0}}=\sqrt{\frac{\Delta}{\Sigma}}\left[c d t-a \sin ^{2} \theta d \varphi\right], \\
\vartheta^{\hat{1}}=\sqrt{\frac{\Sigma}{\Delta}} d r, \\
\vartheta^{\hat{2}}=\sqrt{\Sigma} d \theta, \\
\vartheta^{\hat{3}}=\frac{\sin \theta}{\sqrt{\Sigma}}\left[-a c d t+\left(r^{2}+a^{2}\right) d \varphi\right],
\end{gathered}
$$

where the functions and constants are defined by

$$
\begin{gathered}
\Delta:=r^{2}+a^{2}-2 m r, \quad \Sigma:=r^{2}+a^{2} \cos ^{2} \theta, \\
m:=\frac{G M}{c^{2}} .
\end{gathered}
$$

Direct computation of the charge (4.11) for the tetrad (4.13), (4.15), and (4.16) yields a divergent result. However, we can choose another tetrad with the help of a suitable local Lorentz transformation of the original coframe.

Let us consider the Lorentz transformation described by the matrix $\Lambda=\Lambda_{1} \Lambda_{2} \Lambda_{3}$, where

$$
\Lambda_{1}=\left(\begin{array}{cccc}
1 & 0 & 0 & 0 \\
0 & \cos \varphi & -\sin \varphi & 0 \\
0 & \sin \varphi & \cos \varphi & 0 \\
0 & 0 & 0 & 1
\end{array}\right)
$$

$$
\Lambda_{2}=\left(\begin{array}{cccc}
1 & 0 & 0 & 0 \\
0 & (r / \sqrt{\Sigma}) \sin \theta & \sqrt{\Delta_{0} / \Sigma} \cos \theta & 0 \\
0 & 0 & 0 & 1 \\
0 & \sqrt{\Delta_{0} / \Sigma} \cos \theta & -(r / \sqrt{\Sigma}) \sin \theta & 0
\end{array}\right),
$$

$$
\Lambda_{3}=\left(\begin{array}{cccc}
\sqrt{\Delta_{0} / \Sigma} & 0 & 0 & (a / \sqrt{\Sigma}) \sin \theta \\
0 & 1 & 0 & 0 \\
0 & 0 & 1 & 0 \\
(a / \sqrt{\Sigma}) \sin \theta & 0 & 0 & \sqrt{\Delta_{0} / \Sigma}
\end{array}\right) .
$$

Here $\Delta_{0}=r^{2}+a^{2}$. This Lorentz matrix defines a flat Lorentz connection $\bar{\Gamma}_{\mu}{ }^{\nu}=\left(\Lambda^{-1}\right)_{\gamma}^{\nu} d \Lambda_{\mu}^{\gamma}$. Explicitly, we find:

$$
\begin{aligned}
& \bar{\Gamma}^{\hat{o} \hat{1}}=-\frac{a r \sin ^{2} \theta}{r^{2}+a^{2} \cos ^{2} \theta} d \varphi, \\
& \bar{\Gamma}^{\hat{0} \hat{2}}=-\frac{a \sin \theta \cos \theta \sqrt{r^{2}+a^{2}}}{r^{2}+a^{2} \cos ^{2} \theta} d \varphi, \\
& \bar{\Gamma}^{\hat{0} \hat{3}}=-\frac{a r \sin \theta}{\left(r^{2}+a^{2} \cos ^{2} \theta\right) \sqrt{r^{2}+a^{2}}} d r \\
& +\frac{a \cos \theta \sqrt{r^{2}+a^{2}}}{r^{2}+a^{2} \cos ^{2} \theta} d \theta \\
& \bar{\Gamma}^{\hat{1} \hat{2}}=-\frac{a^{2} \sin \theta \cos \theta}{\left(r^{2}+a^{2} \cos ^{2} \theta\right) \sqrt{r^{2}+a^{2}}} d r-\frac{r \sqrt{r^{2}+a^{2}}}{r^{2}+a^{2} \cos ^{2} \theta} d \theta, \\
& \bar{\Gamma}^{\hat{1} \hat{3}}=-\frac{r \sin \theta \sqrt{r^{2}+a^{2}}}{r^{2}+a^{2} \cos ^{2} \theta} d \varphi, \\
& \bar{\Gamma}^{\hat{2} \hat{3}}=-\frac{\left(r^{2}+a^{2}\right) \cos \theta}{r^{2}+a^{2} \cos ^{2} \theta} d \varphi .
\end{aligned}
$$

One can verify that the flat connection obtained in this way coincides with the Riemannian connection of the original tetrad (4.13), (4.14), (4.15), and (4.16) in the limit of zero $m$, namely $\bar{\Gamma}_{\mu}{ }^{\nu}=\left.\tilde{\Gamma}_{\mu}{ }^{\nu}\right|_{m=0}$. Using this observation, we can straightforwardly recast the charge (4.12) computed for the transformed tetrad into the equivalent form

$$
\widetilde{\mathcal{Q}}^{\prime}\left[\xi, \vartheta^{\prime}\right]=\int_{\partial S} \frac{1}{2 \kappa} \xi^{\alpha}\left(\tilde{\Gamma}^{\mu \nu}-\bar{\Gamma}^{\mu \nu}\right) \wedge \eta_{\alpha \mu \nu} .
$$

This construction actually appears now to be "invariant" under both diffeomorphism and local Lorentz transformations, as $\left(\tilde{\Gamma}^{\mu \nu}-\bar{\Gamma}^{\mu \nu}\right)$ is a covariant quantity. However, the integral obtained is only a result of the choice of a specific frame. Another frame will produce different values for the 
conserved quantities, in general, since it will correspond to different $\bar{\Gamma}$.

The direct evaluation of this integral, for vector fields $\xi$ with constant components $\xi^{i}$ in the coordinate system used in (4.13), (4.14), (4.15), and (4.16), yields finite total conserved charges:

$$
\widetilde{\mathcal{Q}}^{\prime}\left[\xi, \vartheta^{\prime}\right]=\xi^{0} M c^{2}-\xi^{3} \frac{2}{3} M c a .
$$

\section{On the choice of the frame}

As we see, for the tetrad $\vartheta^{\prime \alpha}=\Lambda^{\alpha}{ }_{\beta} \vartheta^{\beta}$ determined by (4.18), (4.19), and (4.20), the conserved charge corresponding to the diffeomorphism generated by the shift along the time coordinate has the usual value $\tilde{\mathcal{Q}}^{\prime}\left[\partial_{t}, \vartheta^{\prime}\right]=M c^{2}$ of the total energy of the configuration. On the other hand, for the vector field along the azimuthal coordinate, we find $\tilde{Q}^{\prime}\left[\partial_{\varphi}, \vartheta^{\prime}\right]=-\frac{2}{3} M c a$. This is proportional to the standard value of the total angular momentum of the Kerr solution, with the coefficient $2 / 3$. In a certain sense, the situation resembles the well-known outcome of the computation of the total mass and angular momentum by using the Komar formulas [21,22] (although in that case the mass appears with a "wrong" coefficient $1 / 2$, and the angular momentum has the standard value $-M c a$ ). More important, however, is the fact that the total mass and angular momentum are both finite for this appropriate choice of the tetrad.

This is analogous to the well-known fact that the computation of the total energy and angular momentum in the framework of the energy-momentum pseudotensor technique necessarily requires a choice of a special class of spacetime coordinates. In the pure tetrad teleparallel approach, the results do not depend on the spacetime coordinates due to use of the explicitly diffeomorphism invariant formalism of exterior forms. However, the choice of the tetrad at spatial infinity becomes an essential aspect of the computation of the conserved quantities.

This was manifested in the above calculation of the conserved charges for the asymptotically flat Kerr configuration. As we have demonstrated, in order to find physically acceptable finite values for the total energy and angular momentum, one needs to select a coframe in an appropriate way. The resulting coframe can be written as follows:

$$
\vartheta^{\prime \alpha}=d \chi^{\alpha}+\left(\sqrt{\Delta}-\sqrt{\Delta_{0}}\right) \phi^{\alpha} .
$$

Here we denoted the quartet of functions

$$
\begin{gathered}
\chi^{\hat{0}}=c t, \quad \chi^{\hat{1}}=\sqrt{\Delta_{0}} \sin \theta \cos \varphi, \\
\chi^{\hat{2}}=\sqrt{\Delta_{0}} \sin \theta \sin \varphi, \quad \chi^{\hat{3}}=r \cos \theta,
\end{gathered}
$$

and introduced the 1-form with the components

$$
\begin{gathered}
\phi^{\hat{0}}=\frac{\sqrt{\Delta_{0}}}{\Sigma} \zeta, \\
\phi^{\hat{1}}=\sin \theta\left(-\frac{r \cos \varphi}{\sqrt{\Delta \Delta_{0}}} d r-\frac{a \sin \varphi}{\Sigma} \zeta\right), \\
\phi^{\hat{2}}=\sin \theta\left(-\frac{r \sin \varphi}{\sqrt{\Delta \Delta_{0}}} d r+\frac{a \cos \varphi}{\Sigma} \zeta\right), \\
\phi^{\hat{3}}=-\frac{\cos \theta}{\sqrt{\Delta}} d r,
\end{gathered}
$$

where $\zeta=c d t-a \sin ^{2} \theta d \varphi$. When $m=0$, this tetrad becomes holonomic, $\left.\vartheta^{\prime \alpha}\right|_{m=0}=d \chi^{\alpha}$. In a sense, we may say that the transformed frame $\vartheta^{\prime}$ describes the "true" gravitational field because, by "switching off" the essential physical parameter $m$, the corresponding field strength $F^{\prime \alpha}=d \vartheta^{\prime \alpha}$ vanishes. In contrast, the 2-form $F^{\alpha}$ does not vanish with $m=0$ for the original coframe (4.13), (4.14), (4.15), and (4.16), which manifests the mixed inertialgravitational nature of this tetrad. Another appealing property of $\vartheta^{\prime}$ is that it is asymptotically holonomic (at spatial infinity), and-what is more important-that its Riemannian connection $\tilde{\Gamma}^{\prime}\left(\vartheta^{\prime}\right)$ vanishes at spatial infinity. Exactly this vanishing of the Riemannian connection makes the convergence of the integral of the conserved charge possible. This completely agrees with our recent observations for the conserved quantities discussed in [1].

Summarizing, the above analysis shows that among all possible tetrads that are formally allowed in teleparallel gravity, there exists a class of tetrads which are characterized, for asymptotically flat gravitational field configurations, by the following properties: they (i) have asymptotically vanishing Riemannian connection, (ii) the total conserved quantities (mass and the angular momentum) are finite for this class of frames. Notice that there remains a freedom to rotate these tetrads by means of a global Lorentz transformation. More generally, as the choice of the tetrad is relevant to the conserved quantities only at the boundary $\partial S$, it is even possible to choose different tetrads, related by a local Lorentz transformation $\Lambda^{\alpha}{ }_{\beta}$ with $d \Lambda^{\alpha}{ }_{\beta} \rightarrow 0$ on $\partial S$ such that the second term on the right-hand side (r.h.s.) of (4.12) is finite (or vanishes).

In the next section, we compare the results obtained within the purely tetrad (quasi-invariant) formulation with an alternative (explicitly invariant) approach to the teleparallel gravity based on the Poincaré gauge theory.

\section{TELEPARALLEL MODEL AS A POINCARÉ GRAVITY WITH CONSTRAINTS}

Teleparallel gravity can be naturally defined as a particular case of Poincaré gauge gravity [20]. Its Lagrangian reads 


$$
\begin{aligned}
V(\vartheta, d \vartheta, \Gamma, d \Gamma, \lambda)= & -\frac{1}{2 \kappa} T^{\alpha} \wedge^{\star}\left({ }^{(1)} T_{\alpha}-2^{(2)} T_{\alpha}-\frac{1}{2}{ }^{(3)} T_{\alpha}\right) \\
& -\lambda^{\alpha}{ }_{\beta} \wedge R_{\alpha}{ }^{\beta} .
\end{aligned}
$$

The last term imposes the vanishing curvature constraint, $R_{\alpha}{ }^{\beta}=d \Gamma_{\alpha}{ }^{\beta}+\Gamma_{\gamma}{ }^{\beta} \wedge \Gamma_{\alpha}^{\gamma}=0$, by means of the Lagrange multiplier 2-form $\lambda^{\alpha}{ }_{\beta}$ (which is antisymmetric in its indices, $\lambda_{\alpha \beta}=-\lambda_{\alpha \beta}$ ). An interesting feature of this model is that besides the diffeomorphism and local Lorentz symmetry, the action is also invariant under the transformation of the Lagrange multiplier

$$
\lambda_{\beta}^{\alpha} \rightarrow \lambda_{\beta}^{\alpha}+D \chi_{\beta}^{\alpha},
$$

with an arbitrary 1-form $\chi^{\alpha}{ }_{\beta}$. This is a direct consequence of the Bianchi identity $D R_{\alpha}{ }^{\beta} \equiv 0$.

For the Lagrangian (5.1) we find $[1,2,23]$

$$
\begin{aligned}
& H_{\alpha}=\frac{1}{2 \kappa} K^{\mu \nu} \wedge \eta_{\alpha \mu \nu}, \quad H_{\beta}^{\alpha}=\lambda_{\beta}^{\alpha}, \\
& \left.\left.\left.E_{\alpha}=e_{\alpha}\right\rfloor V+\left(e_{\alpha}\right\rfloor T^{\beta}\right) \wedge H_{\beta}+e_{\alpha}\right\rfloor R_{\gamma}{ }^{\beta} \wedge H^{\gamma}{ }_{\beta} .
\end{aligned}
$$

Here, $K^{\mu \nu}$ is the contortion 1-form, given by the difference between the Riemannian (Christoffel) and dynamical Riemann-Cartan connections:

$$
K_{\alpha}{ }^{\beta}=\tilde{\Gamma}_{\alpha}{ }^{\beta}-\Gamma_{\alpha}{ }^{\beta} .
$$

The vacuum field equations derived from (5.1) by means of the variation w.r.t. $\vartheta^{\alpha}, \Gamma_{\alpha}{ }^{\beta}$, and $\lambda^{\alpha}{ }_{\beta}$ read, respectively:

$$
\begin{gathered}
D H_{\alpha}-E_{\alpha}=\frac{1}{2 \kappa} \tilde{R}^{\mu \nu} \wedge \eta_{\alpha \mu \nu}=0, \\
D H_{\alpha \beta}+\vartheta_{[\alpha} \wedge H_{\beta]} \equiv D\left(\lambda_{\alpha \beta}-\frac{1}{2 \kappa} \eta_{\alpha \beta}\right)=0, \\
R_{\alpha}{ }^{\beta}=0 .
\end{gathered}
$$

The first Eq. (5.5) is the usual Einstein equation that determines the coframe (modulo local Lorentz transformations). From (5.6) and (5.7) we find the Lagrange multiplier 2-form

$$
\lambda_{\alpha \beta}=\frac{1}{2 \kappa}\left(\eta_{\alpha \beta}+D \chi_{\alpha \beta}\right) .
$$

The 1-form $\chi_{\alpha \beta}$ is arbitrary, reflecting the gauge freedom (5.2). Finally, the constraint Eq. (5.7) determines the Riemann-Cartan connection as a flat connection.

The dynamics of this model is rather degenerate. Besides the freedom of the choice of the Lagrange multiplier, represented by (5.2), the equations for the coframe (tetrad) and for the connection, (5.5) and (5.7), are completely uncoupled. As a result, the flat Riemann-Cartan (i.e., Weitzenböck) connection can be chosen in an arbitrary way, irrespectively of the value of the coframe. Mathematically this is manifested in the possibility of performing independent local Lorentz transformations of the field variables:

$$
\begin{aligned}
& \vartheta^{\prime \alpha}=\Lambda_{I}{ }^{\alpha}{ }_{\beta} \vartheta^{\beta}, \\
& \Gamma_{\alpha}^{\prime}=\left(\Lambda_{I I}^{-1}\right)^{\mu}{ }_{\alpha} \Gamma_{\mu}{ }^{\nu} \Lambda_{I I}{ }^{\beta}{ }_{\nu}+\Lambda_{I I}{ }^{\beta}{ }_{\gamma} d\left(\Lambda_{I I}^{-1}\right)^{\gamma}{ }_{\alpha},
\end{aligned}
$$

with two different Lorentz matrices $\Lambda_{I}{ }^{\alpha}{ }_{\beta} \neq \Lambda_{I I}{ }^{\alpha}{ }_{\beta}$. As a result, we can "rotate" each of the two variables, either $\vartheta^{\alpha}$ or $\Gamma_{\alpha}{ }^{\beta}$, while keeping another one fixed.

\section{A. Conserved invariant charge}

By using the above derivations, the invariant conserved charge for the teleparallel model is computed straightforwardly:

$$
\begin{aligned}
\mathcal{Q}[\xi]= & \frac{1}{2 \kappa} \int_{S}\left[\xi^{\alpha} K^{\mu \nu} \wedge \eta_{\alpha \mu \nu}+\Xi^{\alpha \beta} \eta_{\alpha \beta}\right. \\
& \left.-\left(\mathcal{L}_{\xi} \Gamma^{\alpha \beta}\right) \wedge \chi_{\alpha \beta}\right] .
\end{aligned}
$$

Here $\left.\Xi_{\alpha}{ }^{\beta}=\xi\right\rfloor \Gamma_{\alpha}{ }^{\beta}+\Theta_{\alpha}{ }^{\beta}$, see (3.8). We have also used the identity (A13) of [2]. Recalling that the 1-form $\chi_{\alpha \beta}$ is completely undetermined by the field equations, we conclude that a unique conserved charge can only be defined for symmetric field configurations that satisfy the generalized Killing equation $\mathcal{L}_{\xi} \Gamma^{\alpha \beta}=0$.

Using the identities (A18) of [2] it is direct to prove that, for solutions with vanishing torsion, the Killing equation $\mathcal{L}_{\xi} \vartheta^{\alpha}=0$ implies also that $\mathcal{L}_{\xi} \Gamma_{\alpha}{ }^{\beta}=0$. Therefore, for the Kerr solution we have $\mathcal{L}_{\xi} \Gamma_{\alpha}{ }^{\beta}=0$ for $\xi=$ $\xi^{0} \partial_{t}+\xi^{3} \partial_{\varphi}$, with constant $\xi^{0}$ and $\xi^{3}$. As a result, for such symmetric configurations, the undetermined piece of the Lagrange multiplier disappears from the integral, and the conserved charge reduces to

$$
\mathcal{Q}[\xi]=\frac{1}{2 \kappa} \int_{\partial S}\left[\xi^{\alpha}\left(\tilde{\Gamma}^{\mu \nu}-\Gamma^{\mu \nu}\right) \wedge \eta_{\alpha \mu \nu}+\Xi^{\alpha \beta} \eta_{\alpha \beta}\right] .
$$

Here we used the definition of the contortion (5.4). It is worthwhile to compare this formula with the conserved charge in the pure tetrad formulation (4.25). As we see, the first term (5.11) directly corresponds to (4.25) with the background connection replaced by the dynamical one. There is, however, a second term that does not have counterparts in the pure tetrad framework.

\section{B. Charges for Killing vectors of the Kerr solution}

Let us now compare the computation of the conserved charges in the two frameworks (purely tetrad and Poincaré gravity with constraints) for a specific configuration. We consider again the Kerr solution. Since the conserved charges are well defined only for the generalized Killing vector fields, we now deal with these symmetric configurations.

One can directly check that the Kerr coframe (4.13), (4.14), (4.15), and (4.16) satisfies the symmetry condition $\ell_{\xi} \vartheta^{\alpha}=0$ for the vector field $\xi=\xi^{0} \partial_{t}+\xi^{3} \partial_{\varphi}$, with con- 
stants $\xi^{0}$ and $\xi^{3}$. Now we have to choose the Weitzenböck connection $\Gamma$. It is a flat Riemann-Cartan connection and hence it can always be constructed as $\Gamma_{\mu}{ }^{\nu}=$ $\left(\Lambda^{-1}\right)^{\nu}{ }_{\gamma} d \Lambda^{\gamma}{ }_{\mu}$ with some Lorentz matrix $\Lambda$. It is easy to check that the matrix $\Lambda=\Lambda_{1} \Lambda_{2} \Lambda_{3}$ with the factors defined by (4.18), (4.19), and (4.20) yields an appropriate choice. The resulting components of the Weitzenböck connection are then given by the formulas (4.21), (4.22), (4.23), and (4.24). Finally, we can substitute everything into the formula (5.11) and the direct evaluation of the integrals over the spatial boundary gives the result:

$$
\mathcal{Q}[\xi]=\xi^{0} M c^{2}-\xi^{3} M c a .
$$

We see, the last term in (5.11) turns out to be very important since then the total energy and the total angular momentum have their standard values, $Q\left[\partial_{t}\right]=M c^{2}$ and $\mathcal{Q}\left[\partial_{\varphi}\right]=-M c a$, improving the unusual coefficients in (4.26).

\section{An alternative Lagrangian for teleparallel gravity?}

The comparison of the purely tetrad formulation with the Poincare gauge theory with constraint reveals an interesting observation: both approaches are not ideal. Namely, in the purely tetrad formulation we work with the coframe $\vartheta^{\alpha}$ as the only dynamical variable. It is completely determined (up to local Lorentz rotations) by the field equations. However, the resulting conserved current and charge are not invariant under local Lorentz transformations. Moreover, in general the total conserved quantities are divergent. One should choose a tetrad field that satisfies certain conditions in order to obtain physically meaningful total charges. The situation in the Poincare approach is in a certain sense complementary. Namely, the conserved current and charge are explicitly invariant under both diffeomorphisms and local Lorentz transformations. However, for their computation one needs, besides the tetrad, to know the connection and the Lagrange multiplier. Both of these variables are not determined by the field equations in a unique local Lorentz-covariant way. The arbitrariness in the Lagrange multiplier can be avoided by imposing generalized symmetry conditions on the field configurations. But in the choice of the flat connection there still remains a freedom similar to the freedom of the choice of the coframe in the tetrad formulation.

It seems that one can avoid many (not all, though) difficulties mentioned above by using a different dynamical scheme. Let us outline it here briefly. We can consider the following Lorentz-invariant Lagrangian:

$$
\begin{aligned}
V(\vartheta, d \vartheta, \Gamma, \lambda)= & -\frac{1}{2 \kappa} T^{\alpha} \wedge^{\star}\left({ }^{(1)} T_{\alpha}-2^{(2)} T_{\alpha}-\frac{1}{2}{ }^{(3)} T_{\alpha}\right) \\
& -\lambda^{\alpha}{ }_{\beta} \wedge\left(\Gamma_{\alpha}{ }^{\beta}-\bar{\Gamma}_{\alpha}{ }^{\beta}\right) .
\end{aligned}
$$

Here $\lambda^{\alpha}{ }_{\beta}$ is a Lagrange multiplier 3-form, which imposes the teleparallel constraint by making the connection to reduce to the flat "background" connection $\bar{\Gamma}, R_{\alpha}{ }^{\beta}(\bar{\Gamma})=0$.

The vacuum field equations derived from (5.13) by means of the variation w.r.t. $\vartheta^{\alpha}$, yields the Einstein equation (5.5), as before. Variations w.r.t. $\Gamma_{\alpha}{ }^{\beta}$ and $\lambda^{\alpha}{ }_{\beta}$ lead to

$$
\begin{gathered}
\lambda_{\alpha \beta}+\frac{1}{2 \kappa} D \eta_{\alpha \beta}=0, \\
\Gamma_{\alpha}{ }^{\beta}=\bar{\Gamma}_{\alpha}{ }^{\beta},
\end{gathered}
$$

respectively. The invariant conserved charge for the teleparallel model (5.13) is given by:

$$
\mathcal{Q}[\xi, \vartheta, \bar{\Gamma}]=\frac{1}{2 \kappa} \int_{S} \xi^{\alpha}\left(\Gamma^{\mu \nu}-\bar{\Gamma}^{\mu \nu}\right) \wedge \eta_{\alpha \mu \nu},
$$

which is formally identical to (4.25). Here $\mathcal{Q}[\xi, \vartheta, \bar{\Gamma}]$ is invariant under local Lorentz transformations, but depends on the choice of the flat background connection $\bar{\Gamma}$. The choice of $\bar{\Gamma}$ is, however, equivalent to the choice of a preferred tetrad frame $\vartheta^{\prime}$ in which $\bar{\Gamma}^{\prime}=0$ and $\mathcal{Q}[\xi, \vartheta, \bar{\Gamma}]=\mathcal{Q}\left[\xi, \vartheta^{\prime}, 0\right]$, which then reduces to (4.11) for the frame $\vartheta^{\prime}$, i.e., $\mathcal{Q}\left[\xi, \vartheta^{\prime}, 0\right]=\tilde{Q}\left[\xi\right.$, $\left.\vartheta^{\prime}\right]$. As a consequence of these observations, for the frame $\vartheta$ defined by (4.13), (4.14), (4.15), and (4.16) and the background connection (4.21), (4.22), (4.23), and (4.24), we obtain the finite values (4.26).

\section{DISCUSSION AND CONCLUSIONS}

Our results for the teleparallel gravity models can be interpreted as follows. Tetrad frames differing by a local Lorentz transformation are related to different Lorentz connections. A connection includes both inertial and gravitational effects [24]. While the gravitational effects produced by compact sources vanish asymptotically, the inertial effects can grow up at large distances. This is the case, for example, of the inertial effects in rotating frames. As a consequence, when calculated in a general tetrad frame, the noninvariant conserved quantities can diverge due to the inertial effects carried by that frame. In order to get physically meaningful conserved charges, therefore, it is crucial to choose an appropriate (or preferred) frame, in which the inertial effects are absent, in the sense that the connection vanishes asymptotically. It should be noted that the coordinate counterpart of this property is well known. In fact, it has already been remarked by several authors that, in order to obtain a finite value for the energy of a gravitational system in the usual pseudotensor approach, the integration must be carried out in an asymptotically Minkowskian coordinate system [25,26]. Similar to the choice of the preferred frame, the choice of this coordinate system is crucial in the sense that it does not introduce spurious effects in the calculation of the energy.

The choice of an appropriate frame is ultimately equivalent to the choice of an appropriate connection. This is the idea behind the strategy of using a background connection. 
To understand this point, let us recall that the inertial effects are not covariant. This is quite clear in special relativity, where we know that the inertial effects are not present in the specific class of inertial frames, but do appear in any other class of frames. Being noncovariant, these effects turn out to be represented by a connection [27]. Therefore, if $\Gamma_{\alpha}{ }^{\beta}$ is the connection in a general (orthonormal) frame, before calculating the conserved charges, it is necessary to extract from it all inertial effects connected with the frame, which here are represented by the background connection $\bar{\Gamma}_{\alpha}{ }^{\beta}$ [28]. When we do that, the resulting conserved charge will represent purely gravitational effects, and can consequently be finite. A related open question is whether there exists a frame $\vartheta$ for which (4.11) (or, equivalently, a connection $\bar{\Gamma}$ for which (4.25)) leads to the standard values $\mathcal{Q}[\xi]=M c^{2}$ and $\mathcal{Q}[\xi]=$ $-M c a$ for the Kerr metric.

Summing up, we have developed a general formalism for constructing conserved currents and charges in the gravitational models with quasi-invariant Lagrangians. For such models, the conserved current (3.15) and thus the charge (3.18) are not invariant under local Lorentz transformations even when the field equations are covariant. As an application, we then analyzed an important case of the teleparallel gravity. Another interesting application seems to be the class of models in 3 (or 5 and higher odd dimensions) with topological terms included in the Lagrangian. The case of a 3-dimensional gravity theory of that type was earlier studied in [12], where the corresponding conserved quantities were explicitly derived. The complete analysis of this class of models will be given elsewhere.

\section{ACKNOWLEDGMENTS}

The authors would like to thank FAPESP (Y.N. O. and J. G. P.), CNPq (G. F. R. and J. G. P.), and CAPES for financial support.
[1] Yu. N. Obukhov and G.F. Rubilar, Phys. Rev. D 73, 124017 (2006).

[2] Yu. N. Obukhov and G.F. Rubilar, Phys. Rev. D 74, 064002 (2006).

[3] L. B. Szabados, Living Rev. Relativity 7, 4 (2004); http:// www.livingreviews.org/lrr-2004-4.

[4] H. T. Nieh, J. Math. Phys. (N.Y.) 21, 1439 (1980).

[5] H. T. Nieh and M. L. Yan, J. Math. Phys. (N.Y.) 23, 373 (1982).

[6] F.W. Hehl, W. Kopczyński, J.D. McCrea, and E. W. Mielke, J. Math. Phys. (N.Y.) 32, 2169 (1991).

[7] E. W. Mielke and P. Baekler, Phys. Lett. A 156, 399 (1991); P. Baekler, E. W. Mielke, and F. W. Hehl, Nuovo Cimento B 107, 91 (1992).

[8] T. Kawai, Phys. Rev. D 49, 2862 (1994).

[9] A. A. García, F. W. Hehl, C. Heinicke, and A. Macías, Phys. Rev. D 67, 124016 (2003).

[10] M. Blagojević and M. Vasilić, Phys. Rev. D 68, 104023 (2003).

[11] Yu. N. Obukhov, Phys. Rev. D 68, 124015 (2003).

[12] D. Bak, D. Cagnemi, and R. Jackiw, Phys. Rev. D 49, 5173 (1994).

[13] J. Katz, D. Lynden-Bell, and J. Bicak, gr-qc/0610052.

[14] C. W. Misner, K. S. Thorne, and J. A. Wheeler, Gravitation (Freeman, San Francisco, 1973).

[15] F. Gronwald, Int. J. Mod. Phys. D 6, 263 (1997).
[16] K. Hayashi and T. Shirafuji, Phys. Rev. D 19, 3524 (1979).

[17] Y. Itin, Classical Quantum Gravity 19, 173 (2002).

[18] F. W. Hehl, J. D. McCrea, E. W. Mielke, and Y. Ne'eman, Phys. Rep. 258, 1 (1995).

[19] K. Yano, The Theory of Lie Derivatives and its Applications (North-Holland, Amsterdam, 1955).

[20] Yu. N. Obukhov and J. G. Pereira, Phys. Rev. D 67, 044016 (2003).

[21] A. Komar, Phys. Rev. 113, 934 (1959).

[22] A. Komar, Phys. Rev. 127, 1411 (1962).

[23] Yu. N. Obukhov, Int. J. Geom. Methods Mod. Phys. 3, 95 (2006).

[24] R. Aldrovandi, L. C. T. Guillen, J. G. Pereira, and K. H. $\mathrm{Vu}$, Proceedings of the Albert Einstein Century International Conference, Paris, 2005 (unpublished) [gr-qc/0603122].

[25] V. Faraoni and F. I. Cooperstock, Astrophys. J. 587, 483 (2003).

[26] T. Vargas, Gen. Relativ. Gravit. 36, 1255 (2004).

[27] R. Aldrovandi, P.B. Barros, and J.G. Pereira, Gen. Relativ. Gravit. 35, 991 (2003).

[28] This is the essence of the regularization process introduced in J. W. Maluf, M. V. O. Veiga, and J.F. da RochaNeto, Gen. Relativ. Gravit. (to be published); gr-qc/ 0507122 . 